\title{
Sharp parameter bounds for certain maximal point lenses
}

\author{
Robert Luce, Olivier Sète, Jörg Liesen*
}

April 3, 2014

\begin{abstract}
Starting from an $n$-point circular gravitational lens having $3 n+1$ images, Rhie (2003) used a perturbation argument to construct an $(n+1)$-point lens producing $5 n$ images. In this work we give a concise proof of Rhie's result, and we extend the range of parameters in Rhie's model for which maximal lensing occurs.

We also study a slightly different construction given by Bayer and Dyer $(2007)$ arising from the $(3 n+1)$-point lens. In particular, we extend their results and give sharp parameter bounds for their lens model. By a substitution of variables and parameters we show that both models are equivalent in a certain sense.
\end{abstract}

\section{Introduction}

Gravitational lensing is the deflection of light due to masses located between a light source and an observer, which can produce multiple images of the light source. Standard references describing observations, theory and applications of gravitational lensing include [14, 16, 18].

In this paper we focus on an aspect in the theory of gravitational microlensing, namely the question of maximal lensing, from a mathematical point of view. We consider the case of a single lens plane containing $n$ point masses, and no external shear. Further we assume that the light source is located on the optical axis from the observer through the origin of the lens plane (the center of mass of the point masses). As described, e.g., in [10, 8], the lens equation can be written in this case (using complex numbers) as

$$
0=z_{s}=z-\sum_{k=1}^{n} \frac{m_{k}}{\bar{z}-\bar{z}_{k}}
$$

${ }^{*}$ TU Berlin, Institute of Mathematics, MA 4-5, Straße des 17. Juni 136, 10623 Berlin, Germany (\{luce, sete,liesen\}@math.tu-berlin.de) 
where $z_{s}$ is the position of the light source projected on the lens plane, and $m_{k}$ is the mass of the deflector at the point $z_{k}$ in the lens plane. We point out that several other lens models exist; see, e.g., the recent surveys [11, 12] or [5] (in particular Table 1 which gives an overview of six different models).

Mathematically, the number of lensed images of the source in the above lens equation is equal to the number of (complex) solutions of

$$
\sum_{k=1}^{n} \frac{m_{k}}{z-z_{k}}=\bar{z} .
$$

An important special case of this equation, which we consider in this paper, is the one of $n \geq 3$ equal masses that are located at the vertices of a regular polygon in the lens plane with total mass normalized to unity, i.e., we set $m_{k}=\frac{1}{n}$ and $z_{k}=r e^{i \frac{2 k \pi}{n}}$ for some real number $r>0$ and $k=0,1, \ldots, n-1$. The corresponding lens equation,

$$
R(z)=\bar{z}, \quad \text { where } \quad R(z):=\frac{z^{n-1}}{z^{n}-r^{n}}
$$

has been studied in several publications; see [12, section 2] or [6, sections $2,3]$ for overviews with many pointers to the literature. In particular, Mao, Petters, and Witt [10] showed that this equation has $3 n+1$ solutions if

$$
r<r_{\mathrm{cr}}:=\left(\left(\frac{n-2}{n}\right)^{\frac{n-2}{2}}-\left(\frac{n-2}{n}\right)^{\frac{n}{2}}\right)^{\frac{1}{n}}=\left(\frac{n-2}{n}\right)^{\frac{1}{2}}\left(\frac{2}{n-2}\right)^{\frac{1}{n}},
$$

where $r_{\mathrm{cr}}$ is known as the critical radius. Note that $r_{\mathrm{cr}} \approx 0.7274$ for $n=3$, $r_{\mathrm{cr}}=1 / \sqrt{2} \approx 0.7071$ for $n=4$, and $r_{\mathrm{cr}}$ is strictly monotonically increasing and bounded from above by 1 for $n \geq 4$.

An important result of Khavinson and Neumann [8] states that a rational harmonic function $f(z)-\bar{z}$, with $f(z)$ rational and of degree $n$, may have at most $5(n-1)$ complex zeros (see also [9]). Consequently, any $n$-point lens may have at most $5(n-1)$ images. The lens modeled by $R(z)$ from (11) and with $r<r_{\text {cr }}$ attains this upper bound for the maximal number of images only for $n=3$.

Rhie [15] considered perturbations of $R(z)$ of the form

$$
R_{\varepsilon}(z)=(1-\varepsilon) R(z)+\frac{\varepsilon}{z}=\frac{z^{n}-\varepsilon r^{n}}{z\left(z^{n}-r^{n}\right)},
$$

and indicated that for $n \geq 3,0<\varepsilon<1$ "small enough", and

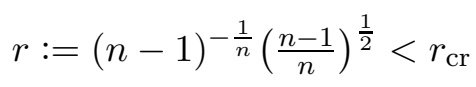

the equation $R_{\varepsilon}(z)=\bar{z}$ has $5 n$ solutions. Since $R_{\varepsilon}(z)$ is of degree $n+1$, Rhie's result implies the existence of maximal point lenses for any $n \geq 3$.

In section 2 we reconsider Rhie's original construction based on the function (2), and we extend Rhie's result from the special value $r$ in (3) to all 
$r \in\left(0, r_{\mathrm{cr}}\right)$. Further we discuss bounds on the eligible weight $\varepsilon$ such that the equation $R_{\varepsilon}(z)=\bar{z}$ describes a maximal point lens. In particular, we derive a sharp bound.

In a related work, Bayer and Dyer [2, 3] intended to close some gaps in the understanding of maximal lensing that remained after [15]. For $n \geq 3$ and $r \in(0,1 / \sqrt{2})$ they derived an upper bound $m_{*}>0$, so that for any $\varepsilon \in\left(0, m_{*}\right)$ the equation

$$
R(z)+\frac{\varepsilon}{z}=\bar{z},
$$

has $5 n$ solutions and thus models a maximal lensing case.

In section 3 we extend their result to a range of radii that is strictly larger than the interval $\left(0, r_{\mathrm{cr}}\right)$, and we give sharp bounds for the mass $\varepsilon$. In the maximal lensing case for the function (2), the maximal eligible mass $\varepsilon$ can be expressed as a function of the radius $r$. This is no longer the case for the function on the left hand side of equation (4), where these two parameters show a more complex interdependence. Despite this interdependence, we present a parameter transformation that allows for an exact characterization of the maximal lensing case for the latter function.

\section{A concise proof of Rhie's construction}

The following result was already shown by Mao, Petters, and Witt [10]. We include a proof for completeness, and because a part of this proof will be used to prove one of our main results below.

Proposition 2.1. Let $n \geq 3$ be given. Depending on the parameter $r$, the solutions to the equation $R(z)=\bar{z}$ with $R(z)$ from (11) are given as follows:

- If $0<r<r_{\mathrm{cr}}$, the equation has exactly $3 n+1$ solutions given by $z=0$ and $3 n$ values of $z$ of the form $r_{1} e^{i \frac{(2 k+1) \pi}{n}}, r_{2} e^{i \frac{(2 k+1) \pi}{n}}$ and $r_{3} e^{i \frac{2 k \pi}{n}}$, $k=0,1, \ldots, n-1$, where $0<r_{1}<r_{2}<1<r_{3}$.

- If $r=r_{\mathrm{cr}}$, the equation has exactly $2 n+1$ solutions given by $z=0$, $\left(\frac{n-2}{n}\right)^{\frac{1}{2}} e^{i \frac{(2 k+1) \pi}{n}}$, and $r_{3} e^{i \frac{2 k \pi}{n}}, k=0,1, \ldots, n-1$, where $r_{3}>1$.

- If $r>r_{\mathrm{cr}}$, the equation has exactly $n+1$ solutions given by $z=0$ and $r_{3} e^{i \frac{2 k \pi}{n}}, k=0,1, \ldots, n-1$, where $r_{3}>1$.

Proof. Obviously, $z=0$ solves $R(z)=\bar{z}$, regardless of the value of $r$. To derive necessary and sufficient conditions for nonzero solutions we use polar coordinates and write $z=\rho e^{i \varphi}$ with $\rho>0$ and $\varphi \in \mathbb{R}$. Then a small manipulation shows that $R(z)=\bar{z}$ can be written as

$$
\left(\rho^{2}-1\right) \rho^{n-2} e^{i n \varphi}=r^{n} .
$$

Since $r>0$, it is necessary that $\rho \neq 1$ and that $e^{i n \varphi}$ is real. Thus we must have either $e^{i n \varphi}=1$ and hence $\rho>1$, or $e^{i n \varphi}=-1$ and hence $0<\rho<1$. 
First consider the case $e^{i n \varphi}=1$. Then $\varphi=\frac{2 k \pi}{n}$ for some $k \in\{0,1, \ldots, n-$ $1\}$, and (5) becomes $f_{+}(\rho):=\rho^{n}-\rho^{n-2}-r^{n}=0$. By Descartes' rule of signs, the polynomial $f_{+}(\rho)$ has exactly one positive root, which we denote by $r_{3}>1$. Hence for all $r>0$ there exist $n$ solutions $r_{3} e^{i \frac{2 k \pi}{n}}, k=0,1, \ldots, n-1$, of the equation $R(z)=\bar{z}$.

Next consider the case $e^{i n \varphi}=-1$. Then $\varphi=\frac{(2 k+1) \pi}{n}$ for some $k \in$ $\{0,1, \ldots, n-1\}$, and (5) becomes $f_{-}(\rho):=\rho^{n}-\rho^{n-2}+r^{n}=0$. The polynomial $f_{-}(\rho)$ has either zero or two positive roots. The only positive root of the derivative $f_{-}^{\prime}(\rho)=\rho^{n-3}\left(n \rho^{2}-(n-2)\right)$ is given by $\left(\frac{n-2}{n}\right)^{\frac{1}{2}} \in(0,1)$. Since $f_{-}(0)=f_{-}(1)=r^{n}>0$, the function $f_{-}(\rho)$ has two distinct roots $r_{1}, r_{2} \in(0,1)$ if and only if

$$
f_{-}\left(\left(\frac{n-2}{n}\right)^{\frac{1}{2}}\right)<0, \quad \text { or, equivalently, } \quad r<\left(\left(\frac{n-2}{n}\right)^{\frac{n-2}{2}}-\left(\frac{n-2}{n}\right)^{\frac{n}{2}}\right)^{\frac{1}{n}}=r_{\mathrm{cr}} .
$$

Thus, for $r \in\left(0, r_{\mathrm{cr}}\right)$ there are two positive roots $0<r_{1}<r_{2}<1$ of $f_{-}(\rho)$, giving $2 n$ solutions $r_{1} e^{i \frac{(2 k+1) \pi}{n}}$ and $r_{2} e^{i \frac{(2 k+1) \pi}{n}}, k=0,1, \ldots, n-1$, of $R(z)=\bar{z}$. For $r=r_{\mathrm{cr}}, \rho=\left(\frac{n-2}{n}\right)^{\frac{1}{2}}$ is a (double) positive root of $f_{-}(\rho)$, giving $n$ solutions $\left(\frac{n-2}{n}\right)^{\frac{1}{2}} e^{i \frac{(2 k+1) \pi}{n}}, k=0,1, \ldots, n-1$. For $r>r_{\text {cr }}$, there are no additional solutions of $R(z)=\bar{z}$.

With an analogous but somewhat more technical proof we now show that in Rhie's construction (2), maximal lensing occurs not only for $r$ from (3), but for all $r \in\left(0, r_{\mathrm{cr}}\right)$.

Theorem 2.2. Let $n \geq 3$ and $r \in\left(0, r_{\mathrm{cr}}\right)$ be given. Denote by $\xi_{1}$ the smallest positive root of the polynomial $(n+2) \xi^{n}-n \xi^{n-2}+2 r^{n}$ and set

$$
\varepsilon_{*}:=\frac{\xi_{1}^{n+2}-\xi_{1}^{n}+r^{n} \xi_{1}^{2}}{r^{n}}
$$

Then $\varepsilon_{*} \in(0,1)$, and for any $\varepsilon \in\left(0, \varepsilon_{*}\right)$, the equation $R_{\varepsilon}(z)=\bar{z}$ has $5 n$ solutions of the form $r_{2} e^{i \frac{(2 k+1) \pi}{n}}, r_{3} e^{i \frac{(2 k+1) \pi}{n}}, r_{4} e^{i \frac{(2 k+1) \pi}{n}}$, and $r_{1} e^{i \frac{2 k \pi}{n}}, r_{5} e^{i \frac{2 k \pi}{n}}$, $k=0,1, \ldots, n-1$, where $0<r_{1}<\sqrt{\varepsilon}<r_{2}<r_{3}<r_{4}<1<r_{5}$. If $\varepsilon=\varepsilon_{*}$ there exist only $4 n$ solutions, and only $3 n$ solutions exist for $\varepsilon>\varepsilon_{*}$.

Proof. Clearly, $z=0$ does not solve $R_{\varepsilon}(z)=\bar{z}$. As in the proof of Proposition 2.1, we now write $z=\rho e^{i \varphi}$, where $\rho>0$ and $\varphi \in \mathbb{R}$. Then $R_{\varepsilon}(z)=\bar{z}$ can be written as

$$
\left(1-\rho^{2}\right) \rho^{n} e^{i n \varphi}=\left(\varepsilon-\rho^{2}\right) r^{n} .
$$

Since the right hand side of (77) is real, $r>0$ (and $\varepsilon \neq 1$ ), it is necessary that $\rho \neq 1, \rho \neq \sqrt{\varepsilon}$, and that either $e^{i n \varphi}=1$ or $e^{i n \varphi}=-1$.

If $e^{i n \varphi}=1$, then $\varphi=\frac{2 k \pi}{n}$ for some $k \in\{0,1, \ldots, n-1\}$. Furthermore, $1-\rho^{2}$ and $\varepsilon-\rho^{2}$ must have same sign, hence either $0<\rho<\sqrt{\varepsilon}$ or $1<\rho$, 
and (7) can be written as $f_{+}(\rho):=\rho^{n+2}-\rho^{n}-r^{n} \rho^{2}+\varepsilon r^{n}=0$. By Descartes' rule of signs $f_{+}(\rho)$ has either zero or two positive roots. From

$$
f_{+}(0)=\varepsilon r^{n}>0, \quad f_{+}(\sqrt{\varepsilon})=\varepsilon^{\frac{1}{n}}(\varepsilon-1)<0, \quad f_{+}(1)=(\varepsilon-1) r^{n}<0,
$$

and $f_{+}(\rho) \rightarrow \infty$ for $\rho \rightarrow \infty$, we see that $f_{+}(\rho)$ indeed has one root $r_{1} \in$ $(0, \sqrt{\varepsilon})$ and one root $r_{5} \in(1, \infty)$. Consequently, for all $r>0$ there exist $2 n$ solutions $r_{1} e^{i \frac{2 k \pi}{n}}$ and $r_{5} e^{i \frac{2 k \pi}{n}}, k=0,1, \ldots, n-1$, of the equation $R_{\varepsilon}(z)=\bar{z}$.

If $e^{i n \varphi}=-1$, then $\varphi=\frac{(2 k+1) \pi}{n}$ for some $k \in\{0,1, \ldots, n-1\}$. Here $1-\rho^{2}$ and $\varepsilon-\rho^{2}$ must have opposite signs. Hence $\sqrt{\varepsilon}<\rho<1$ is necessary, and (77) can be written as $f_{-}(\rho):=\rho^{n+2}-\rho^{n}+r^{n} \rho^{2}-\varepsilon r^{n}=0$. The polynomial $f_{-}(\rho)$ has either one or three positive roots. We will derive necessary and sufficient conditions so that $f_{-}(\rho)$ has three distinct positive roots in the interval $(\sqrt{\varepsilon}, 1)$. We start by noting that the positive roots of the derivative $f_{-}^{\prime}(\rho)=\rho\left((n+2) \rho^{n}-n \rho^{n-2}+2 r^{n}\right)$ are equal to the positive roots of $g(\rho):=$ $(n+2) \rho^{n}-n \rho^{n-2}+2 r^{n}$. From

$$
g^{\prime}(\rho)=n \rho^{n-3}\left((n+2) \rho^{2}-(n-2)\right),
$$

we see that the unique positive root of $g^{\prime}(\rho)$ is $\left(\frac{n-2}{n+2}\right)^{\frac{1}{2}} \in(0,1)$. For all $r \in\left(0, r_{\mathrm{cr}}\right)$ we have $g\left(\left(\frac{n-2}{n+2}\right)^{\frac{1}{2}}\right)<0$. Together with $g(0)=2 r^{n}>0$ and $g(1)=2+2 r^{n}>0$ this shows that $g(\rho)$ and thus $f_{-}^{\prime}(\rho)$ have exactly two positive roots, say $\xi_{1}$ and $\xi_{2}$, with $0<\xi_{1}<\left(\frac{n-2}{n+2}\right)^{\frac{1}{2}}<\xi_{2}<1$. Note that $f_{-}^{\prime}(\rho)$ does not depend on $\varepsilon$, so $\xi_{1}$ and $\xi_{2}$ are independent of $\varepsilon$.

Let us write

$$
f_{-}(\rho)=\rho^{2} p(\rho)-\varepsilon r^{n}, \quad \text { where } \quad p(\rho):=\rho^{n}-\rho^{n-2}+r^{n} .
$$

From the proof of Proposition 2.1 we know that for all $r \in\left(0, r_{\mathrm{cr}}\right)$ the polynomial $p(\rho)$ has exactly two distinct positive roots, say $z_{1}$ and $z_{2}$, where $0<z_{1}<z_{2}<1$. Since $f_{-}(0)=f_{-}\left(z_{1}\right)=f_{-}\left(z_{2}\right)=-\varepsilon r^{n}$, the mean value theorem implies that the only two roots of $f_{-}^{\prime}(\rho)$ satisfy $0<\xi_{1}<z_{1}<\xi_{2}<$ $z_{2}$. From $f_{-}\left(z_{2}\right)<0<f_{-}(1)$ we then see that $f_{-}(\rho)$ has exactly one root $r_{4} \in\left(z_{2}, 1\right)$.

Further, $f_{-}(0)=f_{-}\left(z_{1}\right)<0$ implies that $f_{-}(\rho)$ has two more (distinct) roots if and only if

$$
f_{-}\left(\xi_{1}\right)>0, \quad \text { or, equivalently, } \quad \varepsilon<\frac{\xi_{1}^{2} p\left(\xi_{1}\right)}{r^{n}}=\varepsilon_{*} .
$$

Note that $\varepsilon_{*}>0$ since $p(\rho)>0$ on $\left(0, z_{1}\right)$. Further, $p(\rho)$ is decreasing on $\left(0, z_{1}\right)$, so that $p\left(\xi_{1}\right)<p(0)=r^{n}$, and thus $\varepsilon_{*}<1$. In summary, for all $r \in\left(0, r_{\mathrm{cr}}\right)$ and $\varepsilon \in\left(0, \varepsilon_{*}\right)$, the function $f_{-}(\rho)$ has two more distinct roots $r_{2}, r_{3}$ with $\sqrt{\varepsilon}<r_{2}<\xi_{1}<r_{3}<z_{1}$. Hence, for all $r \in\left(0, r_{\mathrm{cr}}\right)$ and $\varepsilon \in\left(0, \varepsilon_{*}\right)$ there exist $3 n$ solutions $r_{2} e^{i \frac{(2 k+1) \pi}{n}}, r_{3} e^{i \frac{(2 k+1) \pi}{n}}$ and $r_{4} e^{i \frac{(2 k+1) \pi}{n}}$, $k=0,1, \ldots, n-1$, of the equation $R_{\varepsilon}(z)=\bar{z}$. 
On the other hand, if $\varepsilon=\varepsilon_{*}, \xi_{1}$ is a (double) zero of $f_{-}(\rho)$. Then $R_{\varepsilon}(z)=\bar{z}$ has the $2 n$ solutions $\xi_{1} e^{i \frac{(2 k+1) \pi}{n}}$ and $r_{4} e^{i \frac{(2 k+1) \pi}{n}}, k=0,1, \ldots, n-1$, in addition to the $2 n$ solutions corresponding to $r_{1}$ and $r_{5}$. Finally, if $\varepsilon>\varepsilon_{*}$, then only the $n$ solutions $r_{4} e^{i \frac{(2 k+1) \pi}{n}}, k=0,1, \ldots, n-1$, of $R_{\varepsilon}(z)=\bar{z}$ occur (in addition to the $2 n$ solutions corresponding to $r_{1}$ and $r_{5}$ ).

Note that $\varepsilon_{*}$ in (6) is a computable quantity that depends on $n$ and $r$. Fig. 1 displays $\varepsilon_{*}$ for a few values of $n$ and $r \in\left(0, r_{\mathrm{cr}}\right)$. Proposition 2.1 and Theorem 2.2 are illustrated numerically in Fig. 2. In particular, the figure shows that the solutions of $R(z)=\bar{z}$ and $R_{\varepsilon}(z)=\bar{z}$ are located on three and five circles around the origin, respectively.

The bound on $\varepsilon$ given in Theorem 2.2 is sharp, but it is unclear whether the value of $\varepsilon_{*}$ can be parameterized explicitly as a function of $n$ and $r$. Next we give an explicit parameterization of a slightly weaker bound on the eligible mass $\varepsilon$, depending only on $n$ and $r$, such that $R_{\varepsilon}(z)=\bar{z}$ admits the maximal number of solutions.

Corollary 2.3. Let $n \geq 3$ and $r \in\left(0, r_{\mathrm{cr}}\right)$ be given. The equation $R_{\varepsilon}(z)=\bar{z}$ has $5 n$ solutions if

$$
0<\varepsilon<\frac{\zeta_{0}^{n+2}-\zeta_{0}^{n}+r^{n} \zeta_{0}^{2}}{r^{n}}, \quad \text { where } \quad \zeta_{0}:=\frac{n+6}{n+8} r^{\frac{3 n+1}{3 n-6}} .
$$

Proof. We use some notation from the proof of Theorem 2.2, we show that $f_{-}\left(\zeta_{0}\right)>0$ and that $r_{2}<\zeta_{0}<r_{3}$. With some algebraic manipulation one sees that the first assertion is equivalent to (8). Assuming that $f_{-}\left(\zeta_{0}\right)>0$, the second assertion is implied by $\zeta_{0}<\left(\frac{n-2}{n+2}\right)^{\frac{1}{2}}$, which follows from a rather technical calculation (Lemma A.1).

Remark 2.4. An alternative bound can be derived from the approach taken in [2]. There, instead of using $\zeta_{0}$ from above as a "trial point" at which $f_{-}(\rho)$ is positive, the authors choose $\rho_{0}=r^{1+\frac{6}{n}}$. Note that $\rho_{0}<\left(\frac{n-2}{n+2}\right)^{\frac{1}{2}}$ holds for $n \geq 3$ and $r \in\left(0, r_{\mathrm{cr}}\right)$. Applied to our context, the resulting mass bound becomes

$$
0<\varepsilon<\frac{\rho_{0}^{n+2}-\rho_{0}^{n}+r^{n} \rho_{0}^{2}}{r^{n}}=r^{\frac{12}{n}+8}-r^{6}+r^{\frac{12}{n}+2} .
$$

The mass constraint (8) is displayed in Fig. 3 ("lower bound $\zeta_{0}$ ") along with the exact bound given in Theorem 2.2 and the bound from (99) ("lower bound $\left.\rho_{0} "\right)$.

In Theorem 2.2 the bound $r_{\mathrm{cr}}$ on $r$ is suggested by Proposition 2.1. Numerical experiments show that the equation $R_{\varepsilon}(z)=\bar{z}$ can still have $5 n$ solutions for $r$ slightly larger than $r_{\mathrm{cr}}$. We did not try to quantify the exact upper bound on eligible $r$ such that $R_{\varepsilon}(z)=\bar{z}$ has $5 n$ solutions. However, we can show the following. 

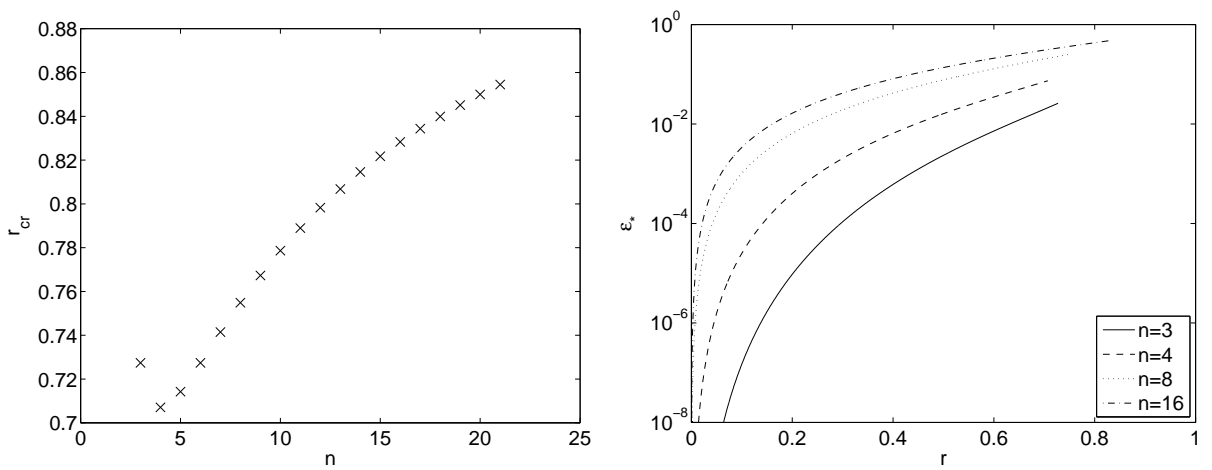

Figure 1: Left: The critical radius $r_{\mathrm{cr}}$ for $3 \leq n \leq 21$. Right: $\varepsilon_{*}$ from (6) for $n=3,4,8,16$ and $r \in\left(0, r_{\mathrm{cr}}\right) ; r_{\mathrm{cr}}(3) \approx 0.7274, r_{\mathrm{cr}}(4) \approx 0.7071, r_{\mathrm{cr}}(8) \approx$ $0.7549, r_{\mathrm{cr}}(16) \approx 0.8283$.
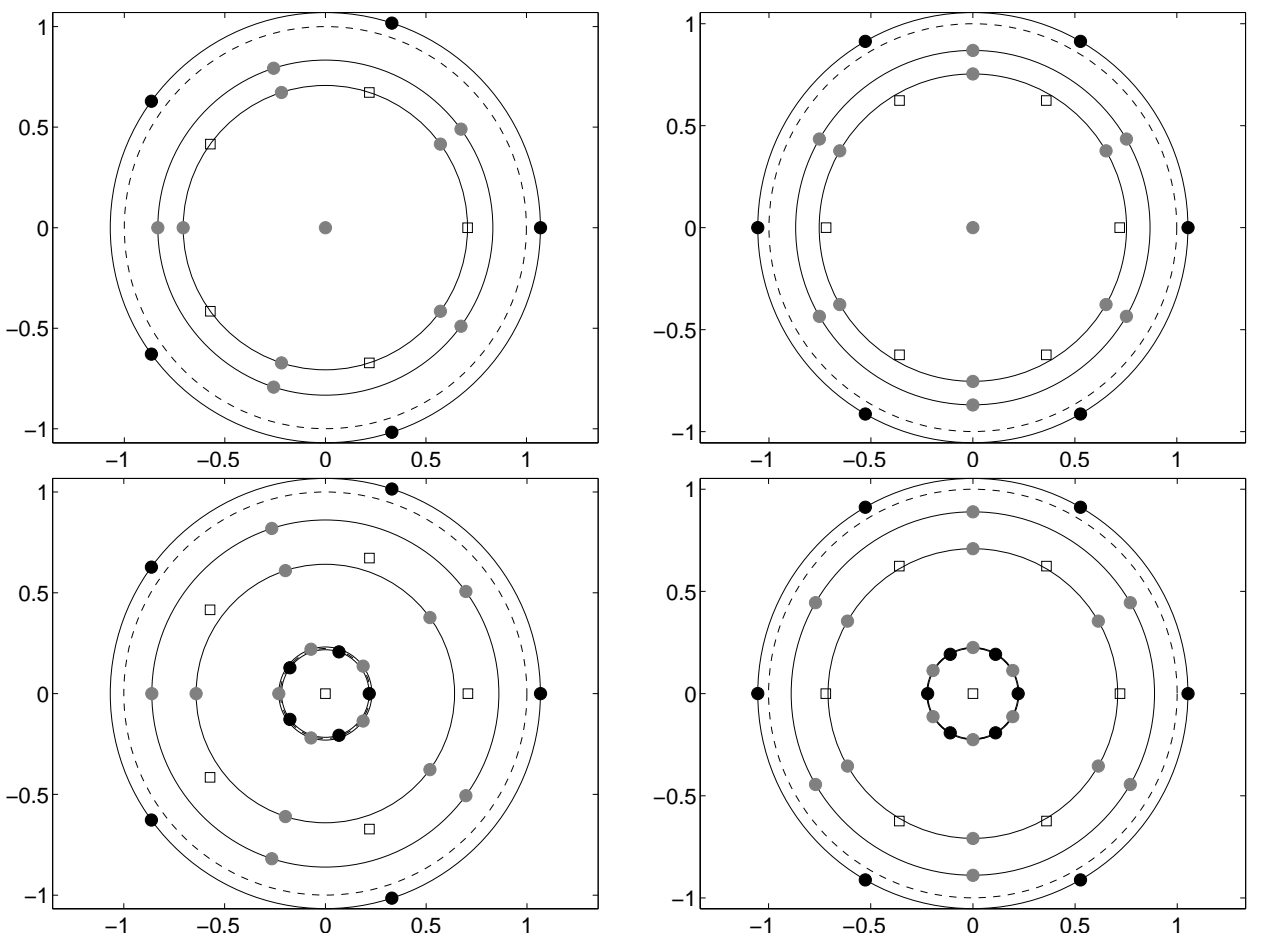

Figure 2: Solutions of $R(z)=\bar{z}$ from (11) (top) and $R_{\varepsilon}(z)=\bar{z}$ from (2) (bottom) for $n=5$ (left) and $n=6$ (right). In both cases we used $\varepsilon=0.05$, and $r$ is chosen as $0.99 \cdot r_{\mathrm{cr}}(n)$ (which is larger than the radius (3D)). The black points correspond to solutions with $e^{i n \varphi}=1$ and the grey points are $z=0$ (top) and the solutions with $e^{i n \varphi}=-1$. Squares indicate the mass points. The dashed circles are the unit circle and the circle of radius $\sqrt{\varepsilon}$ (bottom only). 

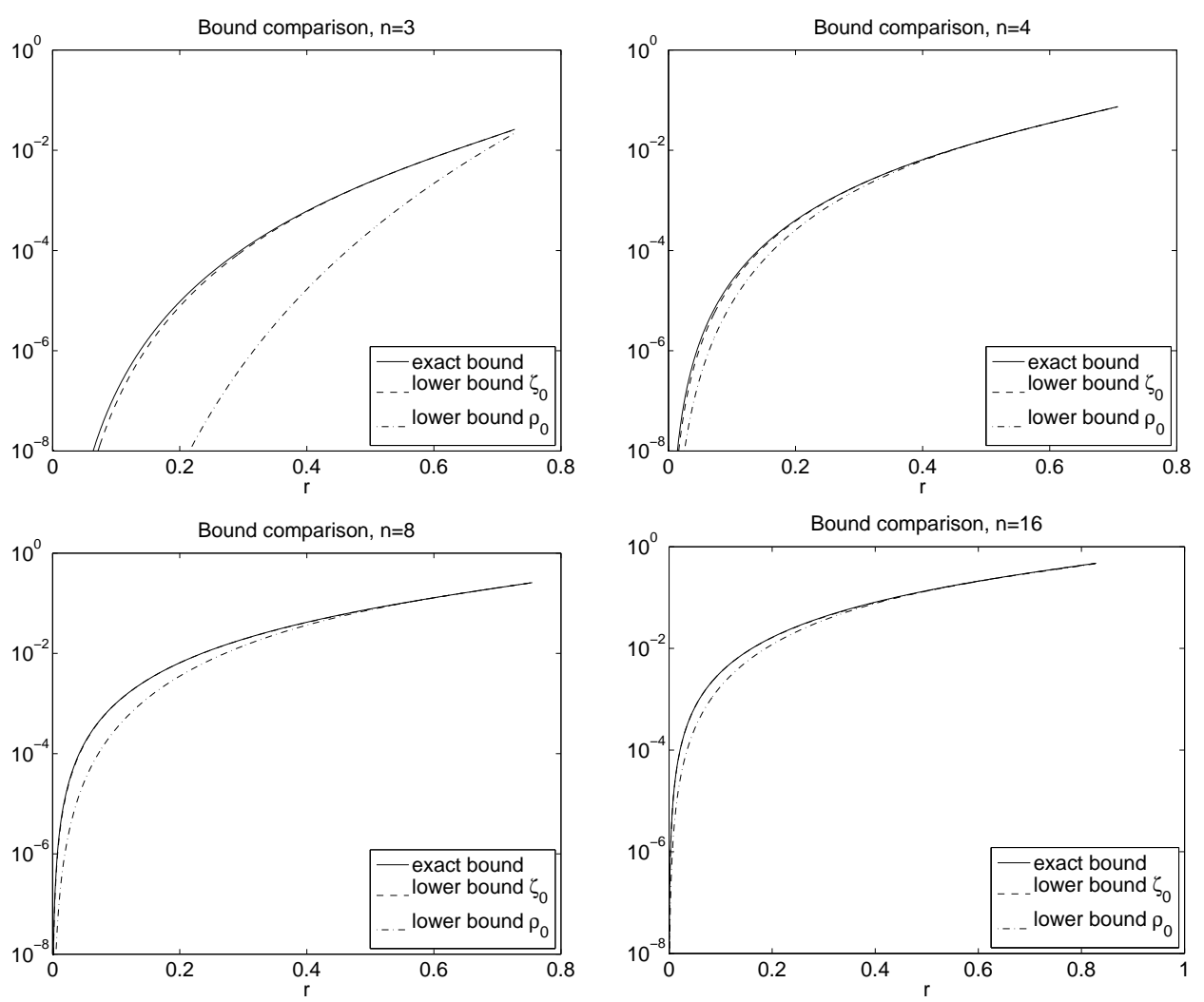

Figure 3: Comparison of mass bounds that imply maximal lensing. For $n=8$ and $n=16$, the exact bound and the lower bound implied by $\zeta_{0}$ are visually indistinguishable. Values below $10^{-8}$ have been clipped.

Corollary 2.5. For any $\varepsilon \in(0,1)$ and $r \geq\left(\frac{n-2}{n+2}\right)^{\frac{n-2}{2 n}}$, the equation $R_{\varepsilon}(z)=\bar{z}$ has exactly $3 n$ solutions.

Proof. In the notation of the proof of Theorem 2.2, we have

$$
g\left(\left(\frac{n-2}{n+2}\right)^{\frac{1}{2}}\right)=\left(\frac{n-2}{n+2}\right)^{\frac{n-2}{2}}\left((n+2) \frac{n-2}{n+2}-n\right)+2 r^{n}=-2\left(\frac{n-2}{n+2}\right)^{\frac{n-2}{2}}+2 r^{n} .
$$

Thus, $r \geq\left(\frac{n-2}{n+2}\right)^{\frac{n-2}{2 n}}$ is equivalent to $g\left(\left(\frac{n-2}{n+2}\right)^{\frac{1}{2}}\right) \geq 0$. Since $g(\rho)$ has its minimum at this point, we have $g(\rho) \geq 0$ for $\rho>0$ with equality at most for $\rho=\left(\frac{n-2}{n+2}\right)^{\frac{1}{2}}$. Now, since $f_{-}^{\prime}(\rho)=\rho g(\rho)$, the function $f_{-}(\rho)$ is strictly monotonically increasing and has only one positive zero. This implies that $R_{\varepsilon}(z)=\bar{z}$ has only $3 n$ solutions. 


\section{$3 \quad$ Extension to additive perturbations}

In Rhie's lens model (2) the perturbation does not alter the total mass of the lens. In fact, since (11) is normalized, the total mass remains normalized to unity after the perturbation.

Another way to model a small mass perturbation of (11) is to increase the total lens mass by placing an additional mass in the origin. This kind of perturbation was considered by Bayer and Dyer [2]. Mathematically, the resulting image configuration of the perturbed lens corresponds to the solutions to the equation $S_{r, \varepsilon}(w)=\bar{w}$, where

$$
S_{r, \varepsilon}(w)=R_{r}(w)+\frac{\varepsilon}{w}=\frac{w^{n-1}}{w^{n}-r^{n}}+\frac{\varepsilon}{w}=\frac{(1+\varepsilon) w^{n}-\varepsilon r^{n}}{w\left(w^{n}-r^{n}\right)} .
$$

Here and in what follows, we write $R_{r}(z)$ and $R_{r, \varepsilon}(z)$ for the functions in (1) and (2) in order to make the dependence on $r$ and $\varepsilon$ explicit.

For notational clarity, we will always use $w$ as the variable in the function (10), as opposed to $z$ which we will reserve for (2). In particular, we will show how to transform solutions from one equation to the other, which necessitates the use of different variable names.

Lemma 3.1. Let $T(z)=\sum_{j=1}^{n} \frac{\sigma_{j}}{z-z_{j}}$ be a rational function and $c>0$. Then

$$
c T(z)=\bar{z} \Leftrightarrow \widetilde{T}(w)=\bar{w},
$$

where $\widetilde{T}(w)=\sum_{j=1}^{n} \frac{\sigma_{j}}{w-w_{j}}, w_{j}=\frac{z_{j}}{\sqrt{c}}$ and $w=\frac{z}{\sqrt{c}}$.

Proof. From

$$
c T(z)=\sqrt{c} \sum_{j=1}^{n} \frac{\sigma_{j}}{\frac{z}{\sqrt{c}}-\frac{z_{j}}{\sqrt{c}}}
$$

we easily see that $c T(z)=\bar{z}$ if and only if $\sum_{j=1}^{n} \frac{\sigma_{j}}{w-w_{j}}=\bar{w}$.

The lemma can be used to map solutions of $R_{r, \varepsilon}(z)=\bar{z}$ and $S_{s, \delta}(w)=\bar{w}$ back and forth, as we will show next.

Proposition 3.2. Let $n \geq 3, r \in\left(0, r_{\mathrm{cr}}\right)$ and $\varepsilon \in(0,1)$ be given. Then

$$
R_{r, \varepsilon}(z)=\bar{z} \Leftrightarrow S_{s, \delta}(w)=\bar{w},
$$

where $s=\frac{r}{\sqrt{1-\varepsilon}}, \delta=\frac{\varepsilon}{1-\varepsilon}$ and $w=\frac{z}{\sqrt{1-\varepsilon}}$.

Proof. Since

$$
R_{r, \varepsilon}(z)=(1-\varepsilon) R_{r}(z)+\frac{\varepsilon}{z}=(1-\varepsilon)\left(R_{r}(z)+\frac{\delta}{z}\right),
$$

Lemma 3.1 shows that $R_{r, \varepsilon}(z)=\bar{z}$ if and only if $S_{s, \delta}(w)=R_{s}(w)+\frac{\delta}{w}=$ $\bar{w}$. 
The correspondence of the parameters $r, s$ and $\varepsilon, \delta$ can be written conveniently as the transformation

$$
(s, \delta)=\Phi(r, \varepsilon)=\left(\frac{r}{\sqrt{1-\varepsilon}}, \frac{\varepsilon}{1-\varepsilon}\right) .
$$

Note that $\Phi$ is bijective with inverse $(r, \varepsilon)=\Phi^{-1}(s, \delta)=\left(\frac{s}{\sqrt{1+\delta}}, \frac{\delta}{1+\delta}\right)$. We will use the transformation $\Phi$ in order to express an analogue to Theorem 2.2 for (10).

Theorem 3.3. Let $n \geq 3$ and $r \in\left(0, r_{\mathrm{cr}}\right)$ be given. The equation $S_{s, \delta}(w)=\bar{w}$ has $5 n$ solutions if and only if $\varepsilon \in\left(0, \varepsilon_{*}\right)$, with $\varepsilon_{*}$ from (6) , and where the parameters $r, \varepsilon$ and $s, \delta$ are connected via (11). These solutions are located on five circles with radii $0<s_{1}<\sqrt{\delta}<s_{2}<s_{3}<s_{4}<\sqrt{1+\delta}<s_{5}$.

Proof. From Proposition 3.2 we know that solutions to $S_{s, \delta}(w)=\bar{w}$ can be mapped to solutions to $R_{r, \varepsilon}(z)=\bar{z}$ via $w=\frac{z}{\sqrt{1-\varepsilon}}=z \sqrt{1+\delta}$ (and vice versa). Using this relation, the assertions follow directly from Theorem 2.2 .

Theorem 3.3 describes parameter pairs $(s, \delta)$ such that maximal lensing occurs. Note that $s$ is not limited by $r_{\mathrm{cr}}$, and that $s$ and $\delta$ are functionally dependent via the transformation (11). In Fig. 4 we compare the range of admissible $(r, \varepsilon)$ pairs with $r<r_{\text {cr }}$ with the transformed pairs $(s, \delta)$. Note also that the upper bound on $\delta$ shown in this figure is sharp for all values of $s$, i.e., maximal lensing cannot happen for values of $\delta$ larger than the ones described in Theorem 3.3 ,

In light of Corollary 2.5, the plots suggest that there is a lower bound on the mass $\delta$, such that maximal lensing happens for values $s>r_{\mathrm{cr}}$. An example for this phenomenon is given in Fig. 5 .

Remark 3.4. By repeating the proof of Theorem 2.2 with respect to (10), another representation of the sharp upper bound on $\delta$ can be obtained as

$$
\delta<\frac{\xi_{1}^{n+2}-\xi_{1}^{n}+s^{n} \xi_{1}^{2}}{s^{n}+\xi_{1}^{n}}
$$

where $\xi_{1}$ is the smallest positive root of $(n+2) \xi^{n}-(1+\delta) n \xi^{n-2}+2 s^{n}$. Note that in contrast to the situation in Theorem 2.2, $\xi_{1}$ now depends on $\delta$, which makes this representation rather difficult to interpret. For convenience, we repeat the proof for this case in Theorem A.2,

We next present an explicit bound for the mass $\delta$, depending only on $s$ and $n$, which implies maximal lensing. The proof follows from Theorem A.2 and by repeating the argument used in Corollary 2.3 .

Theorem 3.5. Let $n \geq 3$ and $s \in\left(0, r_{\mathrm{cr}}\right)$ be given. The equation $S_{s, \delta}(w)=\bar{w}$ has $5 n$ solutions if

$$
\delta<\frac{\zeta_{0}^{n+2}-\zeta_{0}^{n}+s^{n} \zeta_{0}^{2}}{s^{n}+\zeta_{0}^{n}}, \quad \text { where } \zeta_{0}:=\frac{n+6}{n+8} s^{\frac{3 n+1}{3 n-6}} .
$$



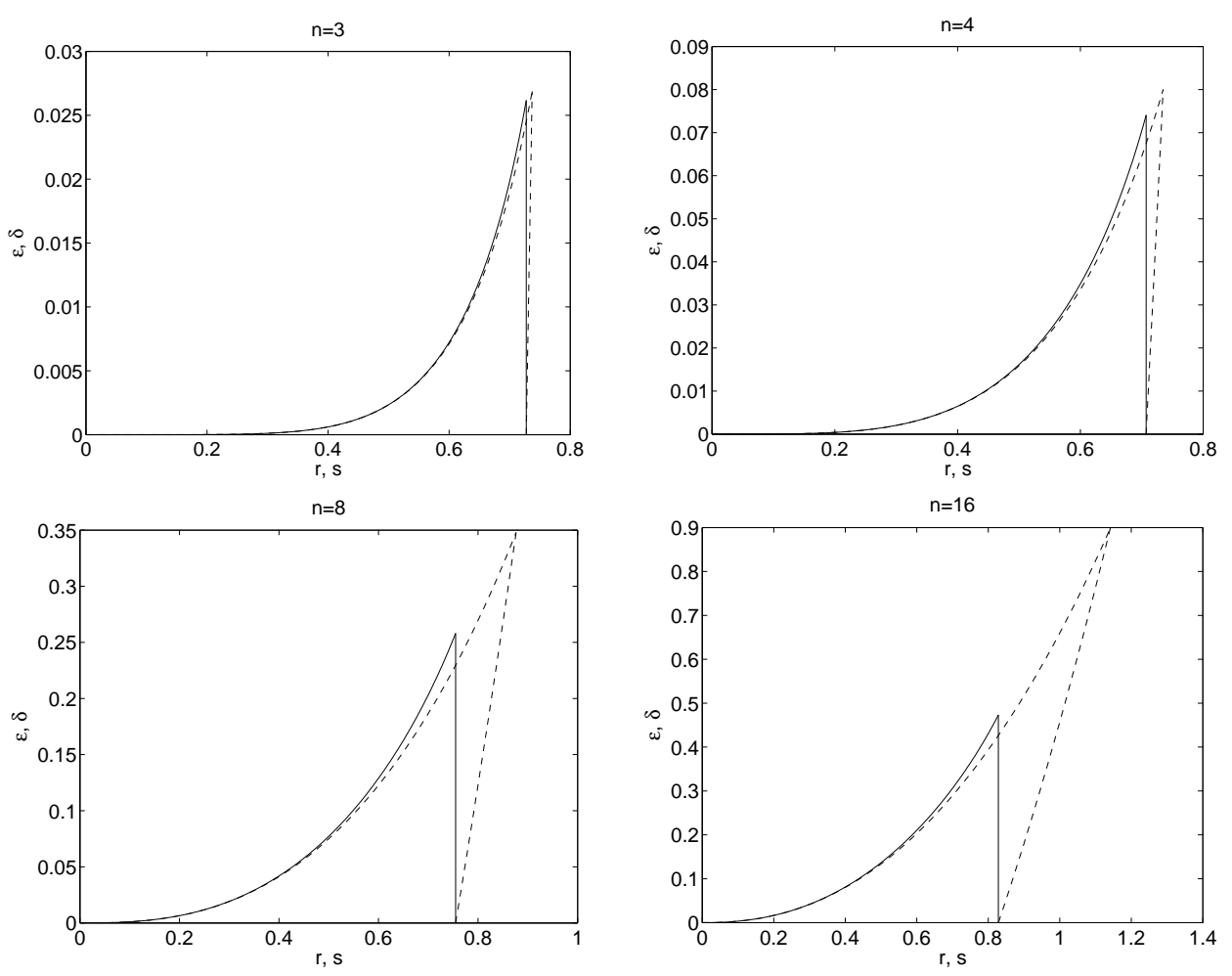

Figure 4: Comparison of parameters $(r, \varepsilon)$ that induce maximal lensing in $R_{r, \varepsilon}(z)=\bar{z}$ with corresponding parameters $(s, \delta)$ for $S_{s, \delta}(w)=\bar{w}$. The region enclosed by the horizontal axis and the solid lines contain all such admissible $(r, \varepsilon)$ pairs, while the transformed $(s, \delta)$ pairs are enclosed by the horizontal axis and the dashed lines.

In [2, equation (7)], another bound is derived as follows. Set $\rho_{0}=s^{1+6 / n}$, then the authors show that if $s<1 / \sqrt{2}$ and

$$
\delta<\frac{\rho_{0}^{n+2}-\rho_{0}^{n}+s^{n} \rho_{0}^{2}}{s^{n}+\rho_{0}^{n}}=s^{2\left(\frac{6}{n}+1\right)}-\frac{s^{6}}{1+s^{6}},
$$

the equation $S_{s, \delta}(w)=\bar{w}$ has $5 n$ solutions. Analogous to Remark 2.4, one can show that the bound is in fact valid for all $s \in\left(0, r_{\mathrm{cr}}\right)$.

In Fig. 6] we compare for $n=3,4,8$ and 16 the exact bound implied by Theorem 3.3 with the bounds (12) and (13). We see that both bounds are asymptotically close to the exact bound, and that the bound based on $\zeta_{0}$ is slightly better than the bound based on $\rho_{0}$.

Finally, we note that maximal lensing may occur even for radii $s>$ $\sqrt{1+\delta} r_{\mathrm{cr}}$. We give the following bound beyond which maximal lensing is impossible. 

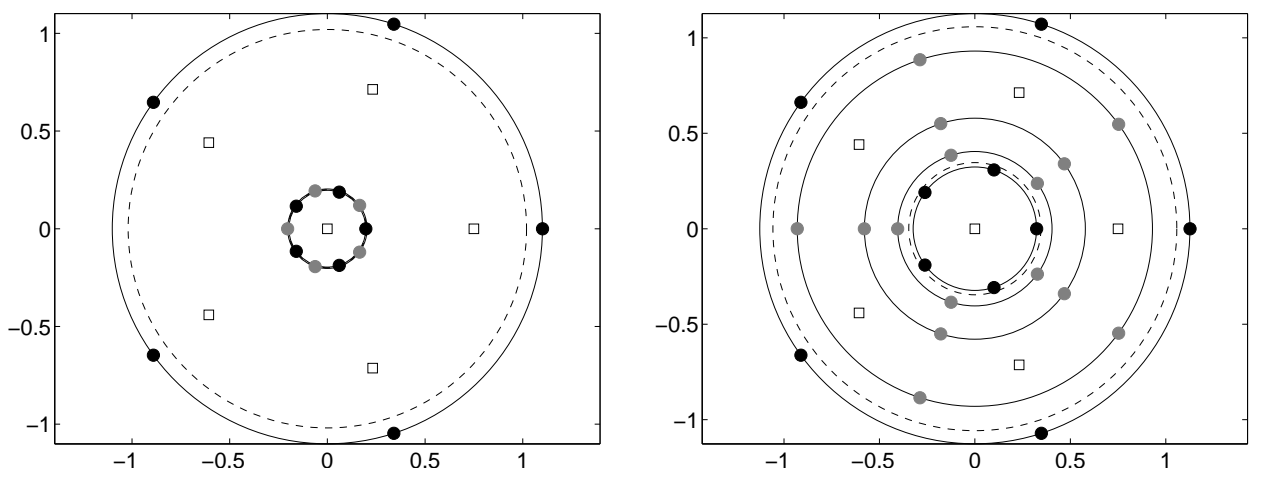

Figure 5: Computed solutions to the equation $S_{s, \delta}(w)=\bar{w}$ with $n=5$, $s=0.75>r_{\text {cr }}$ for masses $\delta_{1}=0.04$ (left) and $\delta_{2}=0.12$ (right). The symbols are as in Fig. 2, The mass $\delta_{1}$ is not sufficiently large for this value of $s$ to induce maximal lensing.

Corollary 3.6. Let $\delta>0$ and $s \geq \sqrt{1+\delta}\left(\frac{n-2}{n+2}\right)^{\frac{n-2}{2 n}}$. Then $S_{s, \delta}(w)=\bar{w}$ has exactly $3 n$ solutions.

Proof. Recall that $R_{r, \varepsilon}(z)=\bar{z}$ has exactly $3 n$ solutions if $r \geq\left(\frac{n-2}{n+2}\right)^{\frac{n-2}{2 n}}$. Since $s=\frac{r}{\sqrt{1-\varepsilon}}=\sqrt{1+\delta} r$, we see by Proposition 3.2 that $S_{s, \delta}(w)=\bar{w}$ has exactly $3 n$ solutions if $s \geq \sqrt{1+\delta}\left(\frac{n-2}{n+2}\right)^{\frac{n-2}{2 n}}$.

Bayer and Dyer [2, 3] considered the situation with three equal point masses arranged at the vertices of an equilateral triangle and a fourth point mass located at its center, i.e. the case $n=3$ in (10). As an application of their bound (13), they consider a maximal lens with total deflector mass of $3.5 \mathrm{M}_{\odot}$, and derive mass constraints on the central mass. Using our exact bound on the central mass in their application (implied by Theorem 3.3) shows that the central mass is bounded in fact by $0.086 \mathrm{M}_{\odot}$ (instead of $0.07 \mathrm{M}_{\odot}$ in [2]).

\section{Conclusions and outlook}

We have given a complete characterization of maximal lensing conditions for Rhie's point lens for radii up to the critical radius $r_{\mathrm{cr}}$. We have presented two bounds on the maximal perturbation mass such that maximal lensing is guaranteed. The first bound is sharp and computable, but given by the roots of certain polynomials, while the other bound is explicitly parameterized and almost as good as the sharp bound.

We have transferred the above mentioned complete characterization to the lens model of Bayer and Dyer, thus extending the known range of radii 

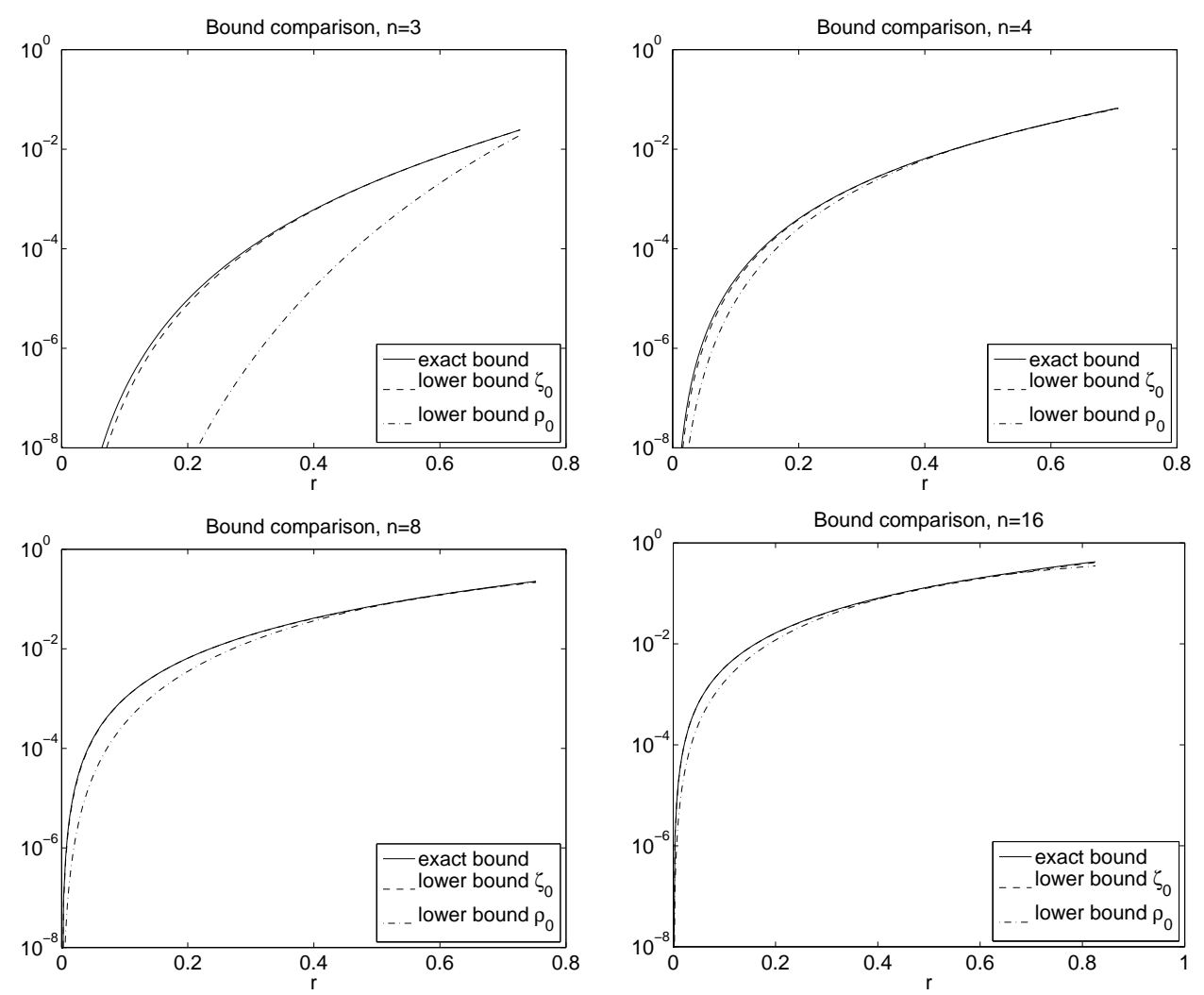

Figure 6: Comparison of the bounds on the eligible masses $\delta$ in the equation $S_{s, \delta}(w)=\bar{w}$ such that maximal lensing occurs. For $n=8$ and $n=16$, the exact bound and the lower bound implied by $\zeta_{0}$ are visually indistinguishable. Values below $10^{-8}$ have been clipped.

for which maximal lensing occurs. We obtained an exact bound on the mass for their model, as well as an approximate bound, which is explicitly given and which improves the previously known one. Our analysis also shows that in the model of Bayer and Dyer maximal lensing is guaranteed to happen even for radii beyond the critical radius.

The methods applied in this paper are specific for the lens modeled by equation (1). Recently we have studied general perturbations of rational harmonic functions of the form $f(z)-\bar{z}$, with $f(z)$ rational, by poles [17. Our results obtained in [17] may also be of interest in the study of the gravitational lensing model considered here. Moreover, we point out that modifications of this model by replacing the point deflectors by spherically symmetric distributed masses, or allowing external shear, have been considered, e.g., in [1, 4, 7, 13]. Whether sharp parameter bounds analogous to ours can be obtained in these models remains a subject of further work. 
Ackowledgements Robert Luce's work is supported by Deutsche Forschungsgemeinschaft, Cluster of Excellence "UniCat".

\section{A Auxiliary proofs}

Lemma A.1. Let $n \geq 3$ and $r \in\left(0, r_{\mathrm{cr}}\right)$ be given. Then $\zeta(r):=\frac{n+6}{n+8} r^{\frac{3 n+1}{3 n-6}}<$ $\left(\frac{n-2}{n+2}\right)^{\frac{1}{2}}$ holds.

Proof. Note that $\zeta(r)$ increases monotonically in $r$, so that $\zeta(r)<\left(\frac{n-2}{n+2}\right)^{\frac{1}{2}}$ for all $r \in\left(0, r_{\mathrm{cr}}\right)$ is equivalent to $\zeta\left(r_{\mathrm{cr}}\right) \leq\left(\frac{n-2}{n+2}\right)^{\frac{1}{2}}$. This last inequality is equivalent to $\left(\frac{n+6}{n+8}\right)^{2} r_{\mathrm{cr}}^{\frac{6 n+2}{3 n-6}} \leq \frac{n-2}{n+2}$. Since $\frac{n+6}{n+8}<1$ it is sufficient to show $r_{\mathrm{cr}}^{6 n+2} \leq\left(\frac{n-2}{n+2}\right)^{3 n-6}$. Inserting the value of $r_{\mathrm{cr}}$ yields $\left(\frac{n-2}{n}\right)^{3 n+1}\left(\frac{2}{n-2}\right)^{6+\frac{2}{n}} \leq$ $\left(\frac{n-2}{n+2}\right)^{3 n-6}$, and after some algebraic manipulation one obtains

$$
\left(1-\frac{2}{n}\right)(n-2)^{-\frac{2}{n}} 2^{6} 4^{\frac{1}{n}}\left(1+\frac{2}{n}\right)^{3 n-6} \leq n^{6} .
$$

The left hand side can roughly be bounded as follows:

$$
\left(1-\frac{2}{n}\right)(n-2)^{-\frac{2}{n}} \cdot 2^{6} \cdot 4^{\frac{1}{n}}\left(1+\frac{2}{n}\right)^{3 n-6} \leq 1 \cdot 1 \cdot 2^{6} \cdot 4^{\frac{1}{3}}\left(\left(1+\frac{2}{n}\right)^{n}\right)^{3} \leq 4^{\frac{1}{3}}(2 e)^{6} .
$$

Now, $4^{\frac{1}{3}}(2 e)^{6} \leq n^{6}$ is equivalent to $n \geq 4^{\frac{1}{18}} 2 e \approx 5.8$, so (14) holds for $n \geq 6$. It is not difficult but tiresome to see by direct calculation that (14) is also valid for $n=3,4,5$.

Theorem A.2. Let $n \geq 3, \delta>0$ and $s \in\left(0, \sqrt{1+\delta} r_{\mathrm{cr}}\right)$ be given and denote by $\xi_{1}:=\xi_{1}(\delta)$ be the smallest positive root of the polynomial $(n+2) \xi^{n}-(1+$ $\delta) n \xi^{n-2}+2 s^{n}$. Then the equation $S_{s, \delta}(w)=\bar{w}$ has $5 n$ solution if and only if

$$
\delta<\frac{\xi_{1}^{n+2}-\xi_{1}^{n}+s^{n} \xi_{1}^{2}}{s^{n}+\xi_{1}^{n}}=: u(\delta) .
$$

If $\delta<u(\delta)$, the $5 n$ solutions have the form $s_{2} e^{i \frac{(2 k+1) \pi}{n}}, s_{3} e^{i \frac{(2 k+1) \pi}{n}}, s_{4} e^{i \frac{(2 k+1) \pi}{n}}$, and $s_{1} e^{i \frac{2 k \pi}{n}}, s_{5} e^{i \frac{2 k \pi}{n}}, k=0,1, \ldots, n-1$, where $0<s_{1}<\sqrt{\delta}<s_{2}<s_{3}<$ $s_{4}<\sqrt{1+\delta}<s_{5}$. If $\delta=u(\delta)$, there exist $4 n$ solutions, and only $3 n$ solutions exist for $\delta>u(\delta)$.

Proof. Obviously, $w=0$ does not solve $S_{s, \delta}(w)=\bar{w}$. As in the proof of Proposition 2.1 we now write $w=\rho e^{i \varphi}$, where $\rho>0$ and $\varphi \in \mathbb{R}$. Then $S_{s, \delta}(w)=\bar{w}$ can be written as

$$
\left(1+\delta-\rho^{2}\right) \rho^{n} e^{i n \varphi}=\left(\delta-\rho^{2}\right) s^{n} .
$$

Since the right hand side of (15) is real and $s>0$, it is necessary that $\rho \neq \sqrt{1+\delta}, \rho \neq \sqrt{\delta}$, and that either $e^{i n \varphi}=1$ or $e^{i n \varphi}=-1$. 
If $e^{i n \varphi}=1$, then $\varphi=\frac{2 k \pi}{n}$ for some $k \in\{0,1, \ldots, n-1\}$. Furthermore, $1+\delta-\rho^{2}$ and $\delta-\rho^{2}$ must have same sign, hence either $0<\rho<\sqrt{\delta}$ or $\sqrt{1+\delta}<\rho$, and (15) can be written as $f_{+}(\rho):=\rho^{n+2}-(1+\delta) \rho^{n}-s^{n} \rho^{2}+$ $\delta s^{n}=0$. From

By Descartes' rule of signs $f_{+}(\rho)$ has either zero or two positive roots.

$$
f_{+}(0)=\delta s^{n}>0, \quad f_{+}(\sqrt{\delta})=-\sqrt{\delta}^{n}<0, \quad f_{+}(\sqrt{1+\delta})=-s^{n}<0,
$$

and $f_{+}(\rho) \rightarrow \infty$ for $\rho \rightarrow \infty$, we see that $f_{+}(\rho)$ indeed has one root $s_{1} \in$ $(0, \sqrt{\delta})$ and one root $s_{5} \in(\sqrt{1+\delta}, \infty)$. Consequently, for all $s>0$ and all $\delta>0$ there exist $2 n$ solutions $s_{1} e^{i \frac{2 k \pi}{n}}$ and $s_{5} e^{i \frac{2 k \pi}{n}}, k=0,1, \ldots, n-1$, of the equation $S_{s, \delta}(w)=\bar{w}$.

Next consider the case $e^{i n \varphi}=-1$, then $\varphi=\frac{(2 k+1) \pi}{n}$ for some $k \in$ $\{0,1, \ldots, n-1\}$. Here $1+\delta-\rho^{2}$ and $\delta-\rho^{2}$ must have opposite signs. Hence $\sqrt{\delta}<\rho<\sqrt{1+\delta}$ is necessary, and (15) can be written as $f_{-}(\rho):=$ $\rho^{n+2}-(1+\delta) \rho^{n}+s^{n} \rho^{2}-\delta s^{n}=0$. The polynomial $f_{-}(\rho)$ has either one or three positive roots. We will derive necessary and sufficient conditions so that $f_{-}(\rho)$ has three distinct positive roots in the interval $(\sqrt{\delta}, \sqrt{1+\delta})$.

The positive roots of the derivative $f_{-}^{\prime}(\rho)=\rho\left((n+2) \rho^{n}-n(1+\delta) \rho^{n-2}+\right.$ $\left.2 s^{n}\right)$ are equal to the positive roots of $g(\rho):=(n+2) \rho^{n}-n(1+\delta) \rho^{n-2}+2 s^{n}$. From

$$
g^{\prime}(\rho)=n \rho^{n-3}\left((n+2) \rho^{2}-(n-2)(1+\delta)\right),
$$

we see that the unique positive root of $g^{\prime}(\rho)$ is $\eta:=\sqrt{1+\delta}\left(\frac{n-2}{n+2}\right)^{\frac{1}{2}} \in(0, \sqrt{1+\delta})$. One can calculate that for all $s \in\left(0, \sqrt{1+\delta} r_{\mathrm{cr}}\right)$ we have $g(\eta)<0$. Together with $g(0)=2 s^{n}>0$ and $g(\sqrt{1+\delta})=2\left(\sqrt{1+\delta}^{n}+s^{n}\right)>0$ this shows that $g(\rho)$ and thus $f_{-}^{\prime}(\rho)$ have exactly two positive roots, say $\xi_{1}$ and $\xi_{2}$, with $0<\xi_{1}<\eta<\xi_{2}<\sqrt{1+\delta}$. Note that $\xi_{1}$ and $\xi_{2}$ depend on $\delta$.

Let us write $f_{-}(\rho)=\rho^{2} p(\rho)-\delta s^{n}$, where $p(\rho):=\rho^{n}-(1+\delta) \rho^{n-2}+s^{n}$. It is easy to see that $p(\rho)$ has two positive roots if and only if $s \in\left(0, \sqrt{1+\delta} r_{\mathrm{cr}}\right)$. These zeros $z_{1}, z_{2}$ satisfy $0<z_{1}<\sqrt{1+\delta}\left(\frac{n-2}{n}\right)^{\frac{1}{2}}<z_{2}<\sqrt{1+\delta}$.

Since $f_{-}(0)=f_{-}\left(z_{1}\right)=f_{-}\left(z_{2}\right)=-\delta s^{n}$, the mean value theorem implies that the two roots of $f_{-}^{\prime}(\rho)$ satisfy $0<\xi_{1}<z_{1}<\xi_{2}<z_{2}$. From $f_{-}\left(z_{2}\right)<0<$ $f_{-}(\sqrt{1+\delta})$ we then see that $f_{-}(\rho)$ has exactly one root $s_{4} \in\left(z_{2}, \sqrt{1+\delta}\right)$.

Further, $f_{-}(0)=f_{-}\left(z_{1}\right)<0$ implies that $f_{-}(\rho)$ has two more (distinct) roots if and only if $f_{-}\left(\xi_{1}\right)>0$, or, equivalently,

$$
\delta<\frac{\xi_{1}^{n+2}-\xi_{1}^{n}+\xi_{1}^{2} s^{n}}{s^{n}+\xi_{1}^{n}}=u(\delta) .
$$

If the above inequality is satisfied, the function $f_{-}(\rho)$ has two more distinct roots $s_{2}, s_{3}$ with $\sqrt{\delta}<s_{2}<\xi_{1}<s_{3}<z_{1}$. Hence, there exist $3 n$ solutions $s_{2} e^{i \frac{(2 k+1) \pi}{n}}, s_{3} e^{i \frac{(2 k+1) \pi}{n}}$ and $s_{4} e^{i \frac{(2 k+1) \pi}{n}}, k=0,1, \ldots, n-1$, of the equation $S_{s, \delta}(w)=\bar{w}$. 
On the other hand, if $\delta=u(\delta), \xi_{1}$ is a (double) zero of $f_{-}(\rho)$. Then $S_{s, \delta}(w)=\bar{w}$ has the $2 n$ solutions $\xi_{1} e^{i \frac{(2 k+1) \pi}{n}}$ and $s_{4} e^{i \frac{(2 k+1) \pi}{n}}, k=0,1, \ldots, n-1$ in addition to the $2 n$ solutions corresponding to $s_{1}$ and $s_{5}$. Finally, if $\delta>$ $u(\delta)$, then only the $n$ solutions $s_{4} e^{i \frac{(2 k+1) \pi}{n}}, k=0,1, \ldots, n-1$, of $S_{s, \delta}(w)=\bar{w}$ occur in addition to the $2 n$ roots corresponding to $s_{1}$ and $s_{5}$.

\section{References}

[1] Jin H. An and N. Wyn Evans. The Chang-Refsdal lens revisited. Monthly Notices of the Royal Astronomical Society, 369(1):317-334, 2006.

[2] Johann Bayer and Charles C. Dyer. Maximal lensing: mass constraints on point lens configurations. Gen. Relativity Gravitation, 39(9):14131418, 2007.

[3] Johann Bayer and Charles C. Dyer. Erratum: Maximal lensing: mass constraints on point lens configurations. Gen. Relativity Gravitation, 41(3):669, 2009.

[4] Johann Bayer, Charles C. Dyer, and Dan Giang. Gravitational lensing and the maximum number of images. Gen. Relativity Gravitation, 38(9):1379-1385, 2006.

[5] Neal Dalal and Jeffrey M. Rabin. Magnification relations in gravitational lensing via multidimensional residue integrals. J. Math. Phys., 42(4):1818-1836, 2001.

[6] C. D. Fassnacht, C. R. Keeton, and D. Khavinson. Gravitational lensing by elliptical galaxies, and the Schwarz function. In Analysis and mathematical physics, Trends Math., pages 115-129. Birkhäuser, Basel, 2009 .

[7] Dmitry Khavinson and Erik Lundberg. Gravitational lensing by a collection of objects with radial densities. Anal. Math. Phys., 1(2-3):139-145, 2011.

[8] Dmitry Khavinson and Genevra Neumann. On the number of zeros of certain rational harmonic functions. Proc. Amer. Math. Soc., 134(4):1077-1085 (electronic), 2006.

[9] Dmitry Khavinson and Genevra Neumann. From the fundamental theorem of algebra to astrophysics: a "harmonious" path. Notices Amer. Math. Soc., 55(6):666-675, 2008. 
[10] S. Mao, A. O. Petters, and H. J. Witt. Properties of point mass lenses on a regular polygon and the problem of maximum number of images. In The Eighth Marcel Grossmann Meeting, Part A, B (Jerusalem, 1997), pages 1494-1496. World Sci. Publ., River Edge, NJ, 1999.

[11] A. O. Petters. Gravity's action on light. Notices Amer. Math. Soc., 57(11):1392-1409, 2010.

[12] A. O. Petters and M. C. Werner. Mathematics of gravitational lensing: multiple imaging and magnification. Gen. Relativity Gravitation, 42(9):2011-2046, 2010.

[13] A. O. Petters and H. J. Witt. Bounds on number of cusps due to point mass gravitational lenses. J. Math. Phys., 37(6):2920-2933, 1996.

[14] Arlie O. Petters, Harold Levine, and Joachim Wambsganss. Singularity theory and gravitational lensing, volume 21 of Progress in Mathematical Physics. Birkhäuser Boston, Inc., Boston, MA, 2001. With a foreword by David Spergel.

[15] S. H. Rhie. n-point Gravitational Lenses with 5(n-1) Images. ArXiv Astrophysics e-prints, May 2003.

[16] P. Schneider, J. Ehlers, and E.E. Falco. Gravitational Lenses. Astronomy and Astrophysics Library. Springer, 1999.

[17] O. Sète, R. Luce, and J. Liesen. Perturbing rational harmonic functions by poles. ArXiv e-prints, March 2014.

[18] Joachim Wambsganss. Gravitational lensing in astronomy. Living Reviews in Relativity, 1(12 (cited on March 19, 2014)), 1998. 